\title{
Force interaction in the expression of causation*
}

\author{
Bridget Copley \\ SFL (CNRS/Paris 8)
}

\author{
Phillip Wolff \\ Emory University
}

\author{
Jason Shepard \\ Emory University
}

\begin{abstract}
Causal meanings in verbs such as cause, enable and prevent have been analyzed as having two components that correspond to two interacting forces or tendencies: one associated with the agent and one with the patient (Talmy 2000; Wolff 2007). In this research we extend a force-dynamic analysis to a wider range of causal and quasi-causal expressions such as lead to, because, and after. The "structural causal pluralism hypothesis" (Copley \& Wolff 2014) is not supported; instead force dynamics is shown to be relevant to expressions throughout syntactic structure. We find that the applicability of the classical force-interaction analysis depends on (i) whether an Agent/Causer is represented in the syntax, and (ii) what kind of causing entity is conceptually represented: either one that generates its own force or one whose force emerges from an interaction with a field in the sense of Copley \& Harley (2015) (e.g., a gravitational field). The latter case, we propose, suggests a criterion for force individuation. This account allows us to identify several classes of causal expressions and to further map out the division of labor between the grammatical and conceptual levels.
\end{abstract}

Keywords: causation, agency, verbs, clausal connectives, force dynamics

\section{Introduction}

The causal meanings of certain verbs have been argued to involve two interacting forces or tendencies (Talmy 2000; Wolff 2007) ${ }^{1}$. One of the forces is associated with the agent and another with the patient, and the kind of interaction between them varies by verb.

For example, the main verb cause is analyzed as describing a kind of scenario, call it CAUSE, in which the agent exerts a force that opposes and is stronger than the

* This work was supported in part by a National Science Foundation grant "Causal illusions and the perception of forces" (BCS-1354088) to Phillip Wolff.

1 The terms force and tendency refer to the same kind of abstract entity; in different cases one term or the other will be more natural, but this does not correspond to an ontological difference. 


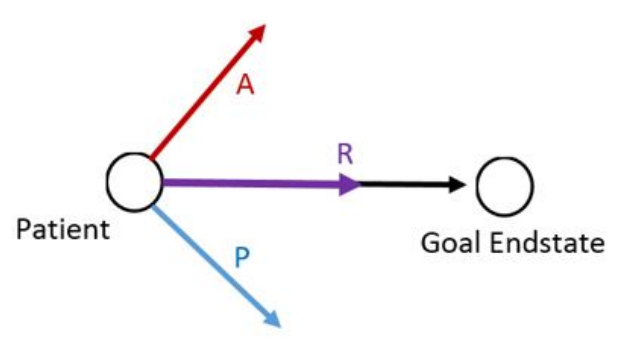

Figure 1 Force-interaction analysis of cause: CAUSE

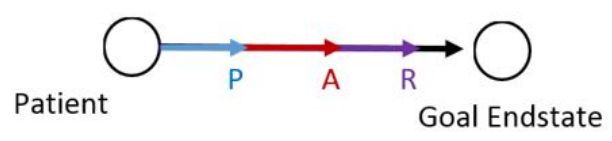

Figure 2 Force-interaction analysis of enable: ENABLE

force exerted by the patient. The force associated with the agent is not in concordance with (not oriented toward the same direction as) the force associated with the patient. Forces can be represented with vectors in an abstract space, such that the strength of the force is represented by the magnitude of the vector, and the abstract direction of the force (i.e., toward or away from a goal endstate) is represented by the spatial direction of the vector. The patient $(\mathrm{P})$ and agent $(\mathrm{A})$ vectors are summed to get a resultant vector $(\mathrm{R})$, as in the representation of CAUSE in Figure 1.

Enable, on the other hand is argued to describe a kind of scenario, call it ENABLE, in which the agent's force is in line with the patient's force, so that the forces associated with the agent and the patient are concordant with each other (Wolff \& Song 2003; Wolff 2007; contra Talmy 2000). This is shown in Figure 2.

Prevent would describe a kind of scenario PREVENT, as in Figure 3, in which the agent's force is oriented away from the goal endstate, and opposes and is stronger than the patient's force, which itself is oriented toward the goal endstate. As in CAUSE scenarios, the force associated with the agent and the patient are not in concordance with each other.

Support for the psychological reality of such meanings has been shown experimentally. For example, Wolff (2007) showed subjects animations of agents and patients with various configurations of their vectors, and asked subjects whether a given configuration corresponded to certain verbs. The key finding was that participants' descriptions of events were indeed sensitive to the two forces in the expected ways. Descriptions involving enable and prevent required that the patient force be 
Force interaction in the expression of causation

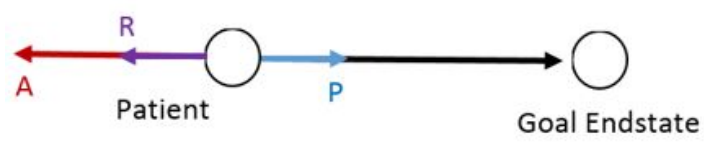

Figure 3 Force-interaction analysis of prevent: PREVENT

oriented towards the final result. Finally, descriptions involving cause and prevent required that there be opposition between the agent and patient forces. Descriptions involving cause and enable required that the resultant vector be directed towards the final result.

We wondered whether other expressions of causation would show similar interactions between agent and patient forces. For example, does because, like cause, necessarily make reference to a CAUSE scenario? Intuitively, this does not seem to be the case: Because seems at least in some circumstances to be compatible with an ENABLE scenario as well (Copley \& Wolff 2014: 55).

To see this, consider (1a) and (1b). Under the assumption that because has the same meaning as cause, the sentence with because in (1b) should also be false. This is because conceptually, the drugs are a mere enabling condition (associated with a force concordant with the Lance Armstrong-associated force) rather than a true cause (which would require Lance Armstrong not to be associated with a force oriented toward winning seven Tours de France). However, (1b) is true. The truth of (1b) seems to indicate that the meaning of because is weaker than that of cause, i.e., it can be used truthfully in both CAUSE and ENABLE scenarios.

a. Drugs caused Lance Armstrong to win seven Tours de France. false

b. Lance Armstrong won seven Tours de France because he took drugs. true

We wondered why because has a weaker meaning. There could be two possible hypotheses for why this might be the case. One possibility explains the difference in meaning by means of two different conceptualizations of causal meanings. The other explains the difference under a single conceptualization of causation (force dynamics), but where the key difference between because and cause is that, unlike cause, because does not grammatically represent an agent force, and therefore cannot explicitly represent force interaction.

\subsection{Structural causal pluralism hypothesis}

The first possibility is that the causal meanings in (1a) and (1b) reflect two different theories of causation. Theories of causation can be divided into two kinds. "Dependency theories" define causation in terms of dependencies between propositions 
(e.g., Lewis 1973; Dowty 1979; Pearl 2000). All that dependency theories require is that the causal factor in some way make a difference in the result, whether by means of causation or enabling. ${ }^{2}$

"Production theories" define causation in terms of the eventualities themselves, without reference to propositions, by means of configurations of forces or transmissions of conserved quantities. Force-dynamic theories of causation are thus production theories. Production theories, unlike dependency theories, can make the distinction between CAUSE and ENABLE scenarios as we have seen above. So, if because were making reference to a dependency theory of causation while cause were making reference to a production theory, this would explain the contrast in (1).

But why would two theories of causation be used for these two different expressions? Copley \& Wolff's (2014) "structural causal pluralism hypothesis" proposes that causal meanings in the cartographic region of phrase structure that deal with events (AspP and below; see, e.g., Ramchand \& Svenonius 2014 and references therein) use force dynamics, while causal meanings of connectives, which take propositional arguments, use dependency causation. This would entail, as desired, that because would fail to distinguish CAUSE and ENABLE scenarios, while cause would distinguish CAUSE and ENABLE scenarios. And in general, other verbs (e.g., leads to, results in) would be expected to behave like cause (because any representation of causation within the verb phrase would have to be force-dynamic), and other clausal connectives would be expected to behave like because (because any representation of causation above AspP would have to be constructed from propositions, via a dependency theory).

\subsection{Forces-everywhere hypothesis}

Another possible explanation for the contrast in (1) - in a sense the null hypothesisis that there are not two theories of causation relevant to the expression of causation in language but only one. Given the need to sometimes distinguish CAUSE from ENABLE scenarios, it follows that the putative single theory must be a production theory, rather than a dependency theory. But it also must be explained why sometimes CAUSE and ENABLE scenarios are not distinguished, as in the case of because.

As can be seen by comparing Figures 1 and 2, the difference between CAUSE and ENABLE scenarios has to do with the relationship between the patient and agent vector. In the case of CAUSE, the agent and patient vectors are not concordant, and in the case of ENABLE, the agent and patient vectors are concordant. The notion of concordance requires the representation of two vectors. Thus, in cases where either the agent or patient force is not specified in the denotation, concordance or its

2 See Dowty (1979: 106-109) for an attempt to work around this limitation within a dependency theory framework. 
Force interaction in the expression of causation

absence can not be determined. So, given that concordance is what distinguishes CAUSE from ENABLE, in such cases, we would not expect CAUSE and ENABLE to be distinguished.

On this hypothesis, there are a number of factors that might be expected to influence whether two forces are represented. Here we will investigate two such factors, one grammatical and one conceptual: first, whether an Agent/Causer role is assigned, and second, whether two forces can be conceptually individuated.

\subsubsection{Assignment of an Agent/Causer role}

Assigning an Agent/Causer role would seem to be necessary in order to have interacting forces in the denotation. Having interacting forces in the denotation would seem to be necessary to distinguish CAUSE from ENABLE scenarios. Thus, a clausal connective such as because would also not be expected to distinguish CAUSE and ENABLE scenarios because it does not assign an Agent/Causer role (not being a verb). We would also expect that verbs that don't assign an Agent/Causer role would not be able to distinguish CAUSE from ENABLE. Two such causal verbs are lead to and result in. For background, note that some nominals refer to entities as in (2a). Others refer to eventualities as in (2b). Although both are grammatically of type e, they have different kinds of referents on the conceptual level, either entities or eventualities (this is along the lines of Roy \& Soare 2013).

(2) a. Entity-referring: the officer, the traffic light

b. Eventuality-referring: the storm, the officer's gesture, the traffic light's changing

The causal predicates lead to and result in-unlike, e.g., cause-do not accept an entity-referring subject at all. Their subject cannot refer to an entity, as in (2a), but rather must refer to an eventuality, as in (2b).

(3) a. \#The officer/\#the traffic light led to/resulted in the woman's walking up to the man.

b. The officer's gesture/the traffic light's changing led to/resulted in the woman's walking up to the man.

The infelicity of (3a) highly suggests that the subject cannot have an Agent/Causer role. Thus, under the forces-everywhere hypothesis, lead to and result in are predicted to not distinguish CAUSE and ENABLE.

On the other hand, the verbs that do allow entity-referring subjects also allow eventuality-describing subjects.

(4) a. The officer allowed/enabled/caused/forced/influenced/made us (to) leave. 
b. The storm/The officer('s gesture)/the traffic light's changing allowed/enabled/caused/forced/influenced/made us (to) leave.

On the forces-everywhere hypothesis, we would expect those in (4) to distinguish CAUSE and ENABLE. The idea is that the verbs in (4) explicitly represent an agent force through the Agent/Causer role (whether filled by an eventuality-referring nominal or an entity-referring nominal). The verbs in (3), however, we expect not to represent an agent force in their denotation. Thus, we would expect these verbs not to be sensitive to a CAUSE/ENABLE distinction, since by hypothesis, to express such a distinction, both the agent and patient vectors must be represented, and in these verbs the agent vector is not represented, because there is no syntactic Agent/Causer role.

\subsubsection{Force generation and the individuation of forces}

On the forces-everywhere hypothesis, another factor that might be expected to influence whether force interaction is represented, and thus whether CAUSE and ENABLE are distinguished, whether two forces are conceptually individuated. This has to do with the way in which the causing force is generated. Some causal verbs, such as make and force, seem to distinguish between an animate force-generating cause, and an inanimate, non-force-generating cause.

(5) The officer/The storm/\#The traffic light made/forced the woman (to) walk up to the man.

An officer and a storm both qualify as force-generating entities as they generate their own force (Wolff, Jeon, Klettke \& Li 2010). On the other hand, a traffic light, while it ultimately has an influence over whether a person crosses the street, does not on its own generate a force that compels the person to cross the street.

But to take a step back, how does the traffic light influence the person if not by generating a force? The answer comes from the notion of a field (Copley \& Harley 2015). A field is an abstract object that, if an entity of the correct kind is placed in it, generates a force on that entity. For instance, the earth's gravitational field is such that if an entity with mass is placed in it, a force on the entity is created. This force, in the case of gravity, is a physical force proportional to the mass of the entity, and directed toward the center of the earth. This abstract notion of field can be extended to fields that generate other physical forces or tendencies, such as the tendency of fruit to ripen when at room temperature, as well as to fields that psychosocially generate forces such as intentions. For example, a social sense of what other people are doing (the field) generates in a person (the entity) an intention to do what other 
people are doing (the tendency). ${ }^{3}$

Intentions can be modeled with vector-like quantities just like physical forces (Talmy 2000; Wolff 2007). In the case of the police officer, a psychosocial force is generated by the officer; this is the agent force. The officer has a certain intention, and because of the officer's social power, the officer's intention has a greater magnitude than the pedestrian's intention. Generally, in the case of a police officer and a pedestrian, if intention is pitted against intention, it is a CAUSE scenario-the officer wins.

Unlike a police officer, a traffic light cannot have an intention. Nonetheless, there is still a way for a traffic light to influence the scene, through the social significance of red and green traffic lights. This social significance is a psychosocial field. That is, when a person who knows what traffic lights mean is placed at a particular spot in an intersection with a red or green light, that configuration creates a certain kind of intention in the person. So even though the traffic light may do essentially the same job as the police officer, there is a difference: When there is a traffic light, there is no imparting of an external force upon the patient. Rather, the traffic light invokes a field that results in the creation of a force on the person. As a consequence, there are not two entities with intentions, but only one, the pedestrian. Thus, in the traffic light condition, there is no Talmian interaction between two tendencies.

The origin of the force associated with the traffic light is in the woman's mind, not in the traffic light itself. So is the origin of her pre-existing intention to cross the street. We propose that the criterion in (6), reminiscent of proposals for event individuation as in Davidson (1969), forbids us from treating two forces whose origin is in a single entity as two forces. Rather, they must be summed and treated as a single force. So while in the officer condition, there is an interaction between two forces, in the traffic light condition, there is only one force.

(6) Force individuation criterion: Two forces that have their origin in the same entity must be summed at the conceptual level together into a single force.

The criterion in (6) is a conceptual, not a grammatical criterion. We hypothesize that such force individuation is a factor in determining whether there is force interaction, and thus whether there is a CAUSE/ENABLE distinction. That is, we expect cases with force-generating causes such as a police officer to involve the representation of two forces, and therefore to distinguish CAUSE and ENABLE.

It's not a priori clear how force individuation and assignment of an Agent/Causer role should interact, but under the forces-everywhere hypothesis, we expect both

3 Talmy analyzes physical forces and intentions with exactly the same kind of theoretical object. Such a view ignores the propositional content of (many) intentions and the lack of propositional content in physical forces (Copley To appear). This difference can be elided here, however. 
Copley, Wolff, and Shepard

of these factors to have an impact on whether CAUSE and ENABLE scenarios are distinguished.

\section{Predictions}

In order to decide between the structural causal pluralism hypothesis and the forceseverywhere hypothesis, we set out to investigate a number of verbal predicates (cause, make, force, influence, enable, allow, lead to, and result in) as well as the clausal connectives because, and, and after. As we have seen, predicates such as cause, make, force ... seem to take an Agent/Causer, while lead to and result in do not take an Agent/Causer. The connectives and and after are of course only "quasi-causal": a causal scenario can be pragmatically inferred even though they themselves do not supply causal meaning.

(7) a. Verbs with Agent/Causer: cause, make, force, influence, enable, allow

b. Verbs without Agent/Causer: lead to, result in

c. Clausal connectives: because, and, after

Thus, if the structural causal pluralism hypothesis is correct, then the following prediction is made:

(8) Prediction (structural causal pluralism hypothesis): Verbs should distinguish CAUSE from ENABLE, and connectives should fail to distinguish CAUSE from ENABLE.

If, on the other hand, the forces-everywhere hypothesis is correct, then the following predictions are made:

(9) Predictions (forces-everywhere hypothesis):

a. Assignment of an Agent/Causer role should be associated with distinguishing CAUSE from ENABLE.

b. Force-generating causes should be associated with distinguishing CAUSE from ENABLE.

\section{Methods}

We tested these hypotheses in an experiment in which people saw animations of various types of interactions. Two main factors were manipulated: (i) the direction of the tendency of the patient (CAUSE vs. ENABLE) and (ii) the manner in which the force was created by the conceptual cause (force-generating entity vs. non-force generating entity). 
Force interaction in the expression of causation

The animations depicted an urban scene in which a woman (the conceptual patient) approached an intersection of two streets. The woman indicated her proclivity to move in a particular direction, by pointing toward one of two possible corners of the intersection. She either pointed toward a corner in which a man was standing or toward a corner that was empty. The conceptual cause in the situation was either a police officer (force-generating) or a traffic light (non-force-generating).

Six-hundred and sixty Amazon's Mechanical Turk workers participated in the experiment.

Three sets of six animations were generated from the animation package 3D Studio Max. Each animation depicted a city scene in which a woman walked to an intersection, paused, and then crossed a street. In one set of animations, a police officer directed the woman to walk in one of two directions. One direction brought the woman to a man standing at one of the corners. The other direction brought the woman to a corner without the man. Similar animations replaced the officer with a traffic light. Each animation lasted 522 frames and was played at approximately 30 frames/second.

Each participant watched each animation, and was asked after each animation to rate several sentences on a scale of $0=$ very unacceptable to $100=$ very acceptable. The sentences used were those in (10) and (11) below:

(10) Officer condition:

a. The officer's gesture caused/enabled/made/forced/allowed/influenced the woman to walk up to the man.

b. The officer's gesture prevented the woman from walking up to the man.

c. The woman walked up to the man because/after the officer gestured.

d. The officer gestured and the woman walked up to the man.

e. The officer's gesture led to/resulted in the woman's walking up to the man.

(11) Traffic light condition:

a. The light's changing caused/enabled/made/forced/allowed/influenced the woman to walk up to the man.

b. The light's changing prevented the woman from walking up to the man.

c. The woman walked up to the man because/after the light changed.

d. The light changed and the woman walked up to the man.

e. The light's changing led to/resulted in the woman's walking up to the man. 


\section{Officer causer}

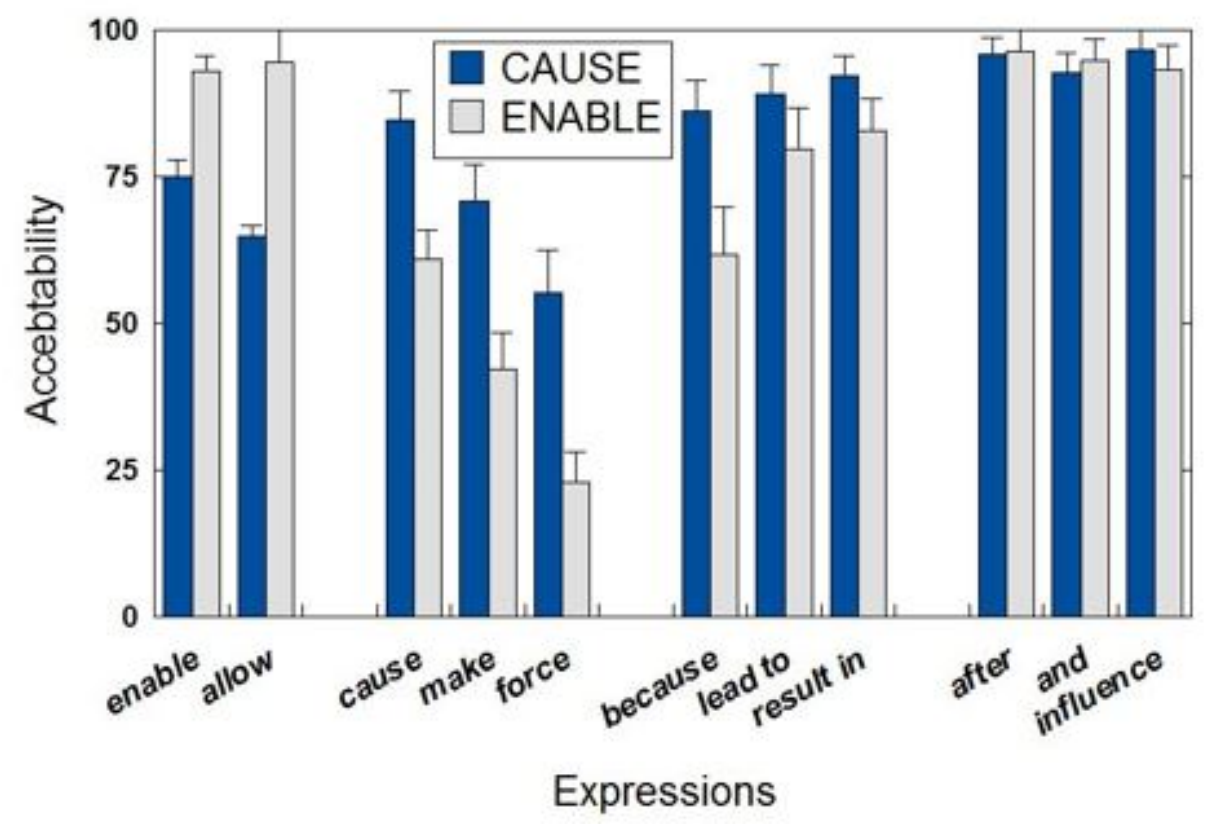

Figure 4 Results: Officer (force-generating cause)

\section{Results}

Recall that we were interested in whether causal expressions were sensitive to two characteristics of the scenarios: first, the direction of the patient vector compared to the agent vector, and second, the kind of cause (the force-generating officer versus the non-force-generating traffic light). If a causal expression were sensitive to the patient vector, it would show a difference in its acceptability rating between the CAUSE scenario and the ENABLE scenario. If a causal expression were sensitive to the kind of cause, it would show a difference in its acceptability rating in the officer condition versus its acceptability rating in the traffic light condition.

The results ${ }^{4}$ are shown graphically in Figures 4 and 5. To summarize: Only certain verbs were always sensitive to both the direction of the patient vector and the kind of cause. These were the verbs cause, make, force, and allow. Other expressions were sensitive to the direction of the patient vector only when the cause

4 In what follows, we do not present the results for prevent and the PREVENT scenario. Both behaved as predicted by Wolff's (2007) theory: that is, prevent was universally judged unacceptable in CAUSE and ENABLE scenarios, and nothing other than prevent was judged acceptable in PREVENT scenarios. 


\section{Traffic light causer}

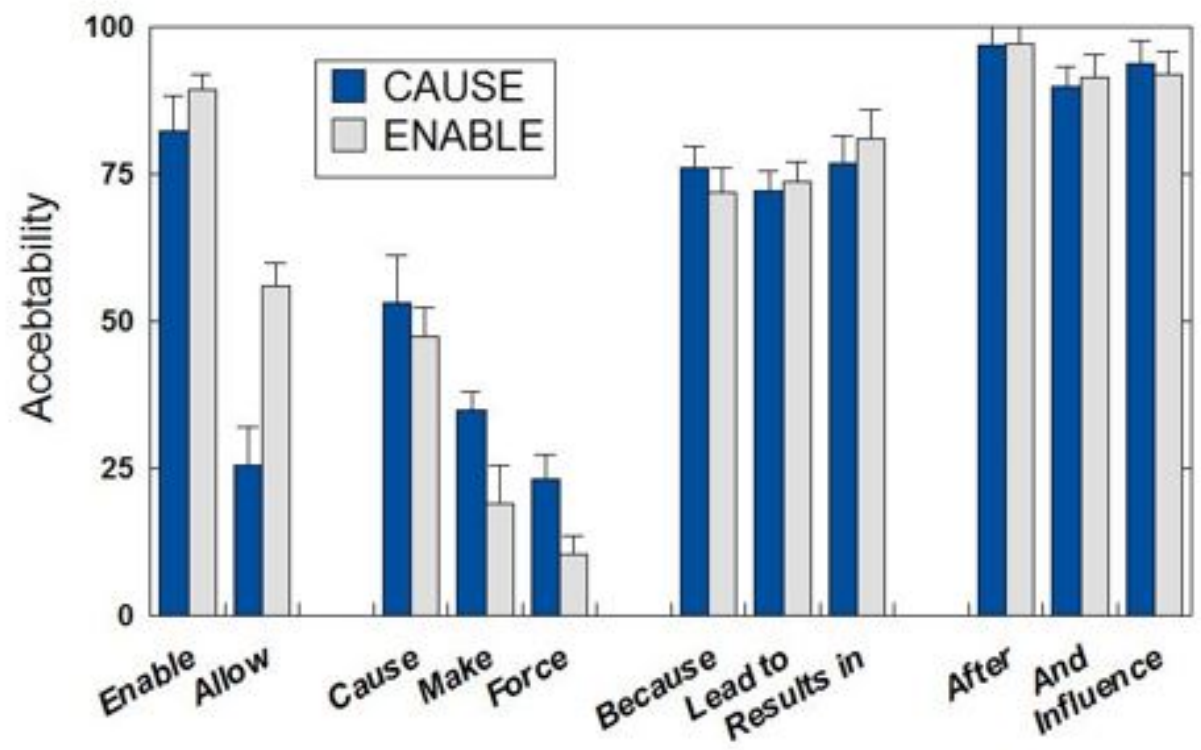

Expressions

Figure 5 Results: Traffic light (non-force-generating cause)

was force-generating, but otherwise were not sensitive to the kind of cause: because, lead to, result in, and enable. Finally, some expressions were sensitive to neither: the quasi-causal connectives after and and, and the verb influence. These generalizations are shown below in Table 1 .

\section{Discussion}

Our results provided evidence against the causal pluralism hypothesis and evidence for the forces-everywhere hypothesis. We will treat these in turn, and then discuss implications for the division of labor at the grammar-cognition interface, as well the denotations of both the cases where the denotation seems to explicitly refer to force interaction (cause, make, force, enable) and the other cases, where the denotation seems not to explicitly refer to force interaction. 
Copley, Wolff, and Shepard

\begin{tabular}{c|c|c|c} 
& $\begin{array}{c}\text { CAUSE } \neq \text { ENABLE } \\
\text { always }\end{array}$ & $\begin{array}{c}\text { CAUSE } \neq \text { ENABLE } \\
\text { only if cause is fg }\end{array}$ & $\begin{array}{c}\text { CAUSE }=\text { ENABLE } \\
\text { always }\end{array}$ \\
\hline $\begin{array}{c}\text { fg cause } \neq \\
\text { non-fg cause }\end{array}$ & $\begin{array}{c}\text { cause, make, } \\
\text { force, allow }\end{array}$ & & \\
\hline fg cause $=$ & & $\begin{array}{c}\text { lead to, result in, } \\
\text { enable, because }\end{array}$ & influence, after, and \\
non-fg cause & & & \\
\hline
\end{tabular}

Table 1 Results categorized

\subsection{Structural causal pluralism hypothesis not supported}

The structural causal pluralism hypothesis predicts that causal meanings in the cartographic region of phrase structure that deal with events (AspP and below) represent causation using force-dynamic force interaction as in Figures 1 - 3, while causal meanings of connectives, which take propositional arguments, use dependency causation. This would suggest that verbs should distinguish CAUSE and ENABLE, while connectives should not.

The prediction made by this hypothesis, given in (8), is not borne out by our results. First, verbs did not always distinguish CAUSE from ENABLE scenarios: influence never did, and enable, lead to and result in did only when the cause was force-generating. Second, the connective because distinguished CAUSE and ENABLE when the cause was force-generating. Our results, therefore, do not support the structural causal pluralism hypothesis.

\subsection{Forces-everywhere hypothesis supported}

Since the structural clausal pluralism prediction was not supported, we should revert to the null hypothesis where only one theory of causation is used throughout phrase structure. As discussed above, this single theory has to be a production theory, of which a force-dynamic theory is one kind. Our force-dynamic theory makes the predictions in (9) above, namely that assignment of an Agent/Causer role, as well as whether the cause is force-generating or non-force-generating, should be associated with a distinction between CAUSE and ENABLE scenarios.

Both predictions were supported by the evidence. Assigning an Agent/Causer role turned out to be a necessary condition for always distinguishing CAUSE from ENABLE. It was not a sufficient condition, as enable and influence do not behave like cause, make, force and allow; but recall that there was never any expectation that it would be a sufficient condition, since representing the agent vector is not the only factor determining whether two forces can be represented in the denotation. 
For example, the patient vector also needs to be represented.

Not surprisingly, the quasi-causal connectives and and after proved always insensitive to the CAUSE/ENABLE distinction, as would be expected given that their denotations do not seem to represent causation at all. Also in this group is influence, which does on the other hand seem to explicitly have a causal meaning and even an Agent/Causer, suggesting that something else is preventing the grammatical representation of force interaction; see Section 5.5 below for a suggestion as to what.

There was also a class of causal expressions that distinguished CAUSE and ENABLE only when the cause was force-generating (the officer). This class consists of enable, the non-agentive verbs lead to, and result in, and the connective because. The existence of this class supports the second prediction of the forces-everywhere hypothesis concerning force individuation: the force-generating cause was associated with distinguishing CAUSE and ENABLE.

Thus, the forces-everywhere hypothesis is supported by our results: the two predicted factors, namely assignment of an Agent/Causer and whether the cause is force-generating, have the predicted impact on whether CAUSE and ENABLE scenarios are distinguished.

\subsection{The division of labor between grammar and cognition}

These results also shed light on the division of labor between the grammatical level (i.e., the denotation) and the conceptual level. They do so by indicating how the aforementioned factors - one grammatical, one conceptual-interact.

A common assumption is that the denotation $i s$ the representation of conceptual structure. This assumption is held explicitly by cognitive linguists such as Talmy, and is often (though not always) held implicitly by formal semanticists. Our results here suggest that this assumption is not correct.

The reason is that, if the assumption were correct, we would not expect to see a mismatch between the two factors. In particular, we would not expect to see force interaction (a distinction between CAUSE and ENABLE) in the cases where there is no Agent/Causer represented. But we do see exactly this, namely in the case of lead to, result in, enable, and because. And in fact we see evidence of force interaction exactly when there is a force-generating cause, i.e., for a conceptual reason-an effect that does not show up in the cases where we would suspect both forces are grammatically represented, namely cause, make, force and allow.

Taken in its entirety, this pattern means we have to distinguish between the representation of forces at the grammatical level from the representation of forces at the conceptual level. Whenever a force is represented at the grammatical level (in the denotation), it is necessarily represented at the conceptual level as well. If the force is represented grammatically, it can be individuated (even contrary to the 
force individuation criterion in (6)), and compared to another force. However, the behavior of the class consisting of lead to, result in, enable and because shows us that there can be forces represented, and compared, at the conceptual level even though they are not explicitly represented in the denotation, and that if such merely conceptually-represented forces are not individuated by the denotation, they are subject to the force individuation criterion.

\subsection{Force interaction in the denotation}

If cause, make, force, and allow really do represent two forces in their denotations, how do they do this? We propose the following claim:

(12) Only verbal predicates can express the interaction of two forces in their denotation.

Plausible support for this claim can be constructed from a relatively minimal extension of existing theory of "flavors" of the causal head v (Folli \& Harley 2005).

As background, there has been much work separating the causal meaning from the lexical root meaning in the structure of verbs. Kratzer (1996) and Marantz (1997) use evidence from phrasal idioms to argue that agents do not appear to really be arguments of the verb they appear with. The argument is that particular patients can trigger a particular idiomatic reading of a verb, as in (13), while particular agents rarely if ever do.

(13) a. throw a baseball, throw support behind a candidate, throw a boxing match

b. take a book from the shelf, take a bus to New York, take a nap

c. kill a cockroach, kill a conversation, kill an evening watching TV

These facts are unexpected if agents are arguments of their verbs, as in (14a). However, if the causal meaning is understood as something (syntactically) distinct from the lexical meaning, as in (14b), then these facts make sense: the agent is not syntactically close enough to the lexical root meaning to interact with it. Rather, it is the causal head $\mathrm{v}$ that takes the meaning of the lexical root and specifies the agent's causing of the eventuality, represented by the Davidsonian argument e (Davidson 1967).

(14) a. old analysis: $\lambda \times \lambda e$. throw(John, baseball, e)

b. v: $\lambda x \lambda$ e . agent(John)(e) \& throw(baseball)(e)

This classic proposal for $\mathrm{v}$, though it does not mention forces, provides a basis for understanding how agent and patient forces might be represented in verbal semantics, 
and why it might be impossible to represent in the denotation both the agent and patient force with causal connectives. If we interpret Davidsonian arguments as forces (f) instead of events (e), as proposed by Copley \& Harley (2015) in their semantics for $\mathrm{v}$, we can see how this plays out.

Copley \& Harley, following Folli \& Harley's (2005) categorization of meanings associated with v into different "flavors" of v, e.g., $\mathrm{v}_{d o}$ and $\mathrm{v}_{\text {become }}$, adapt Dowty's (1979) BECOME operator (as in (15a)) to be their $\mathrm{v}_{\text {become }}$. Added is the idea that an Agent/Causer-initiated force, arising in an initial situation init(f) in which not-p is true, leads to the final situation $f i n(\mathrm{f})$ in which $\mathrm{p}$ is true (as in (15b)).

(15) a. Dowty 1979: BECOME $\Phi$ is true at t iff $\Phi$ is true at $t$ and $\Phi$ is false at t -1 .

b. Copley \& Harley's (2015) $\llbracket \mathrm{v}_{\text {become }} \rrbracket=\lambda \mathrm{p} \lambda \mathrm{f} . \neg \mathrm{p}(\operatorname{init}(\mathrm{f})) \& \mathrm{p}(\operatorname{fin}(\mathrm{f}))$

So, in (15b), a force provokes a change in whether $\mathrm{p}$ is true between the initial situation and the final situation. The Agent/Causer itself is added by a higher Voice head introducing the SOURCE of the force, understood literally as the source of the energy. The predicate $\pi$ is a predicate of forces.

$$
\llbracket \text { Voice }_{\text {active }} \rrbracket=\lambda \pi \lambda \mathrm{x} \lambda \mathrm{f} . \pi(\mathrm{f}) \& \operatorname{SOURCE}(\mathrm{x}, \mathrm{f})
$$

This makes the denotation of the whole Voice phrase as in (17), where $p$ is the predicate and $\mathrm{x}$ is the Agent/Causer (though strictly speaking, $\mathrm{p}$ and $\mathrm{x}$ would be saturated with lexical items by this point):

$$
\llbracket \text { VoiceP } \rrbracket=\lambda \mathrm{f} . \neg \mathrm{p}(\operatorname{init}(\mathrm{f})) \& \mathrm{p}(f i n(\mathrm{f})) \& \operatorname{SOURCE}(\mathrm{x}, \mathrm{f})
$$

But note that only one force is mentioned: the Agent/Causer force. To represent the diagrams in Figures 1 and 2, at least two forces need to be mentioned, and their result needs to be calculated. In particular the force that represents the patient's tendency must be mentioned, in addition to the Agent/Causer force. However, mentioning the patient directly in the denotation of the $\mathrm{v}$ head would seem to be problematic, as $\mathrm{v}$ does not have a direct syntactic relationship with the patient, and therefore should not have a direct semantic relationship with it either.

Copley \& Harley's framework suggests a way out of this difficulty. They propose (2015: 148) a notion of what we might call extraneous forces, namely, forces that are extraneous to the force exerted by the Agent/Causer. To represent these, the notion of a force being "in" a situation is needed, represented with the symbol $<$.

An extraneous force $f$ ' in the initial situation of $f$ (i.e., in Talmian terms, the ground - the situation that is causally prior to the application of $\mathrm{f}$ ) represents a force that could come from the patient, but is not required to. Really, all we know is that it does not come from the agent. The reason why is that an extraneous force $f$ ' that exists in the initial situation of $f$ cannot be identical to $f$. The initial situation of $f$ is 
causally prior to $\mathrm{f}$, and $\mathrm{f}$ ' is in the initial situation of $\mathrm{f}$; therefore $\mathrm{f}$ ' is causally prior to $f$. Thus, f' can serve as a patient vector - though it could also be a force arising from anything in the initial situation, whether or not it is mentioned in the sentence.

Furthermore, there is a way to represent the resultant vector, by an extraneous force that is in the final situation of $f$. The final situation of $f$ depends on the application of $\mathrm{f}$; it is causally subsequent to $\mathrm{f}$. Thus, any force $\mathrm{f}$ " that is in the final situation of $\mathrm{f}$ is one that has already taken $\mathrm{f}$ into account.

We can thus propose that certain causal verbs have their own Talmian "flavors" of $v$, each of which represent three forces: the Agent/Causer force $f$, the "patient" force f', and the resultant force f", as in (18). The only difference between (18a) and $(18 b)$ is the negation in (18a).

$$
\begin{aligned}
& \text { a. } \mathrm{v}_{\text {opposed }}: \lambda \mathrm{p} \lambda \mathrm{f} . \exists \mathrm{f}^{\prime}<(\operatorname{init}(\mathrm{f})):\left[\neg \mathrm{p}\left(\operatorname{fin}\left(\mathrm{f}^{\prime}\right)\right)\right] \& \exists \mathrm{f} "<(f i n(\mathrm{f})):\left[\mathrm{p}\left(f i n\left(\mathrm{f}^{\prime \prime}\right)\right)\right] \\
& \text { b. } \mathrm{v}_{\text {aligned }}: \lambda \mathrm{p} \lambda \mathrm{f} . \exists \mathrm{f}^{\prime}<(\operatorname{init}(\mathrm{f})):\left[\mathrm{p}\left(\operatorname{fin}\left(\mathrm{f}^{\prime}\right)\right)\right] \& \exists \mathrm{f} "<(f i n(\mathrm{f})):\left[\mathrm{p}\left(\operatorname{fin}\left(\mathrm{f}^{\prime \prime}\right)\right)\right]
\end{aligned}
$$

In (18a) $, \exists \mathrm{f}^{\prime}<(\operatorname{init}(\mathrm{f})):\left[\neg \mathrm{p}\left(\operatorname{fin}\left(\mathrm{f}^{\prime}\right)\right)\right]$ indicates that there is an extraneous force in the ground (i.e., in the situation $\operatorname{init}(\mathrm{f}))$ that, if unopposed, would result in a final situation where $\neg \mathrm{p}$ was true. For $(18 \mathrm{~b}) \exists \mathrm{f}^{\prime}<(\operatorname{init}(\mathrm{f}))$ : [ $\left.\mathrm{p}\left(\operatorname{fin}\left(\mathrm{f}^{\prime}\right)\right)\right]$ indicates that there is an extraneous force in the ground (i.e., in the situation init(f)) that, if unopposed, would result in a final situation where $\mathrm{p}$ was true. In both (18a) and (18b), the second conjunct $\exists \mathrm{f}$ " < $(f i n(\mathrm{f})):\left[\mathrm{p}\left(f i n\left(\mathrm{f}^{\prime}\right)\right)\right]$ indicates that there is a resultant force f" resulting from the agent force $\mathrm{f}$ (i.e., in the situation $f(\mathrm{f})$ ) such that, if $\mathrm{f}$ " is unopposed, it results in a final situation where $\mathrm{p}$ is true. The denotations in (18a) and (18b) thus parallel the diagrams given in Figure 1 and Figure 2 respectively. Thus, cause, make and force would be expected to use $\mathrm{v}_{\text {opposed }}$ and allow would be expected to use $\mathrm{v}_{\text {aligned }}$.

If these flavors of $\mathrm{v}$ are the right way to think about cause, make, force, and allow, we can understand both why not all verbs are in that class, and why no clausal connectives are in that class. For the verbs, not all verbs would be expected to have these particular flavors of $\mathrm{v}$. While every $\mathrm{v}$ head's semantics would be some variation on (15b), not every $v$ head's semantics would be one of the particular variations given in (18). For the connectives, since they would not be expected to have a $v$ head at all, the Dowtian semantics in (18a) and (18b) would not be possible. They would thus not be able to compare both forces explicitly, which is as desired.

\subsection{Lack of force interaction in the denotation}

Before we conclude, we will say a few speculative words about the denotations of the causal expressions where there seems to be no force interaction in the denotation.

For verbs without an Agent/Causer such as lead to and result in (and possibly also enable, though it seems to at least have an agentive reading), it's very tempting 
to propose that these also use the flavors of $\mathrm{v}$ in (18), but without an Agent/Causer introduced by a Voice head. Lead to and result in would use $\mathrm{v}_{\text {opposed }}$ while enable would (might) use $\mathrm{v}_{\text {aligned }}$. If this were true, though, contrary to expectation from our results, a causing force would be represented, and in fact even an interaction between forces would be represented-however, the causing force would not explicitly be associated with an Agent/Causer, and that might be significant. For reasons of space we cannot address the consequences of such a proposal here, but it seems worth investigating.

The verb influence would be one of the verbs that has a different flavor of $\mathrm{v}$ from those in (18). Influence cannot use the denotation in (18a) because although it assigns an Agent/Causer, there is no evidence of force interaction, as it is never sensitive to the CAUSE/ENABLE distinction. Instead, influence probably describes a force that changes the degree to which a proposition holds of an entity. In that case, it is likely that what is missing is a representation of the patient vector. So, influence would need a flavor of $\mathrm{v}$ similar to $\mathrm{v}_{\text {become }}$ as in (15b), and would have a Voice head to introduce the Agent/Causer as in (15b); but unlike $\mathrm{v}_{\text {become }}$, it would not itself specify which direction the change is in. We will not develop this idea further here, but see Copley \& Harley (Manuscript) for the use of both force and degree arguments for $\mathrm{v}$ denotations.

Finally, the denotations of the quasi-causal connectives and and after must be distinguished from that of because. All of these being clausal connectives, their conjuncts are propositional (predicates of situations), but recall that and and after never distinguish CAUSE from ENABLE, while because distinguishes CAUSE from ENABLE when the cause is force-generating.

The conjuncts in and and after might be expected to refer to two different situations, one in each conjunct. After, in particular, would specify a temporal relation between two situations, but not a causal relation. Pragmatically, however, for either and or after, the two different situations could be understood as two causally-related situations $s_{n}$ and $s_{n+1}$ (see, e.g., Moeschler 1989). On the other hand, because would explicitly represent two causally-related situations, perhaps as in (19), where pred(s) picks out the causally preceding situation of a situation s (Copley \& Harley 2015):

$$
\llbracket \text { because } \rrbracket=\lambda \mathrm{p} \lambda \mathrm{q} \lambda \mathrm{s} \cdot \mathrm{p}(\operatorname{pred}(\mathrm{s})) \& \mathrm{q}(\mathrm{s})
$$

This difference would capture the fact that because, in contrast to and and after, shows sensitivity to the CAUSE/ENABLE distinction in the case of a forcegenerating cause. This is because for because, we need to understand one situation (the officer's gesture) as causing the next situation (the woman's walking up to the man). This would plausibly trigger a conceptual-level comparison of forces in $\operatorname{pred}(\mathrm{s})$. On the other hand, with and and after, we need not view their two conjuncts' 
Copley, Wolff, and Shepard

situations as causally related, so the comparison is not necessarily triggered.

\section{Conclusion}

The goal of this research was to investigate the application of the force-interaction analysis to a wider range of causal expressions. Two hypotheses were considered: either that force dynamics was relegated to verbal expressions, or that force dynamics was relevant throughout the structure. The second hypothesis proved to be supported, and representation of force interaction was seen to depend on two factors: (i) whether an Agent/Causer is represented in the syntax, and (ii) what kind of causing entity is conceptually represented: either one that generates its own force or one whose force emerges from an interaction with a field. Our findings suggested a partial independence between conceptual representation of forces and grammatical representation of forces. The conceptual-level facts hinged on a criterion of force individuation that requires different forces to have different origins. An addition to the inventory of flavors of $v$ allowed us to account for why only (certain) verbs can represent force interaction at the grammatical level, and we speculatively proposed denotations for the cases without grammatical force interaction.

\section{References}

Copley, Bridget. To appear. Force dynamics. In Rob Truswell (ed.), Oxford Handbook of Event Structure, Oxford University Press.

Copley, Bridget \& Heidi Harley. 2015. A force-theoretic framework for event structure. Linguistics and Philosophy 38(2). 103-158.

Copley, Bridget \& Heidi Harley. Manuscript. Reifiying energy and change in the verb phrase using forces and degrees. Unpublished CNRS and University of Arizona ms.

Copley, Bridget \& Phillip Wolff. 2014. Theories of causation should inform linguistic theory and vice versa (Oxford Studies in Theoretical Linguistics vol. 52). In Bridget Copley \& Fabienne Martin (eds.), Causation in grammatical structures, 11-57. Oxford University Press.

Davidson, Donald. 1967. The logical form of action sentences. In Nicholas Rescher (ed.), The logic of decision and action, 81-95. University of Pittsburgh Press.

Davidson, Donald. 1969. The individuation of events. In Nicholas Rescher (ed.), Essays in Honor of Carl G. Hempel, vol. 24 Synthese Library, 216-234. Springer.

Dowty, David. 1979. Word Meaning and Montague Grammar: The Semantics of Verbs and Times in Generative Semantics and in Montague's PTQ. Reidel.

Folli, Raffaella \& Heidi Harley. 2005. Flavors of v. In Paula Kempchinsky \& Roumyana Slabakova (eds.), Aspectual Inquiries, 95-120. Springer. 
Force interaction in the expression of causation

Kratzer, Angelika. 1996. Severing the external argument from its verb. In Johan Rooryck \& Laurie Zaring (eds.), Phrase Structure and the Lexicon, 109-137. Springer.

Lewis, David. 1973. Causation. The Journal of Philosophy 70. 556-567.

Marantz, Alec. 1997. No escape from syntax: Don't try morphological analysis in the privacy of your own lexicon. University of Pennsylvania Working Papers in Linguistics 4(2). 201-225.

Moeschler, Jacques. 1989. Pragmatic connectives, argumentative coherence and relevance. Argumentation 3(3). 321-339.

Pearl, Judea. 2000. Causality: Models, Reasoning, and Inference. Cambridge University Press.

Ramchand, Gillian \& Peter Svenonius. 2014. Deriving the functional hierarchy. Language Sciences 46. 152-174.

Roy, Isabelle \& Elena Soare. 2013. Event-related nominals. In Gianina Iordăchioaia, Isabelle Roy \& Kaori Takamine (eds.), Categorization and Category Change, 123-152. Cambridge Scholars Publishing.

Talmy, Leonard. 2000. Towards a Cognitive Semantics, Volume 1: Concept Structuring Systems. Cambridge, MA: MIT Press.

Wolff, Phillip. 2007. Representing causation. Journal of Experimental Psychology: General 136(1). 82.

Wolff, Phillip, Ga-hyun Jeon, Bianca Klettke \& Yu Li. 2010. Force creation and possible causers across languages. In Barbara Malt \& Phillip Wolff (eds.), Words and the Mind: How Words Capture Human Experience, 93-110. Oxford University Press.

Wolff, Phillip \& Grace Song. 2003. Models of causation and the semantics of causal verbs. Cognitive Psychology 47(3). 276-332.

Bridget Copley

CNRS - SFL

59-61 rue Pouchet

75017 Paris, France

bridget.copley@cnrs.fr

Jason Shepard

479 Psychology Building

36 Eagle Row

Emory University

Atlanta, GA 30322, USA

jason.s.shepard@emory.edu
Phillip Wolff

479 Psychology Building

36 Eagle Row

Emory University

Atlanta, GA 30322, USA

pwolff@emory.edu 